\title{
Electrophysiological correlates of anterior cingulate function in a go/no-go task: Effects of response conflict and trial type frequency
}

\author{
SANDER NIEUWENHUIS and NICK YEUNG \\ Princeton University, Princeton, New Jersey \\ and \\ WERY VAN DEN WILDENBERG and K. RICHARD RIDDERINKHOF \\ University of Amsterdam, Amsterdam, The Netherlands
}

\begin{abstract}
Neuroimaging and computational modeling studies have led to the suggestion that response conflict monitoring by the anterior cingulate cortex plays a key role in cognitive control. For example, response conflict is high when a response must be withheld (no-go) in contexts in which there is a prepotent tendency to make an overt (go) response. An event-related brain potential (ERP) component, the N2, is more pronounced on no-go than on go trials and was previously thought to reflect the need to inhibit the go response. However, the N2 may instead reflect the high degree of response conflict on no-go trials. If so, an N2 should also be apparent when subjects make a go response in conditions in which nogo events are more common. To test this hypothesis, we collected high-density ERP data from subjects performing a go/no-go task, in which the relative frequency of go versus no-go stimuli was varied. Consistent with our hypothesis, an N2 was apparent on both go and no-go trials and showed the properties expected of an ERP measure of conflict detection on correct trials: (1) It was enhanced for low-frequency stimuli, irrespective of whether these stimuli were associated with generating or suppressing a response, and (2) it was localized to the anterior cingulate cortex. This suggests that previous conceptions of the no-go N2 as indexing response inhibition may be in need of revision. Instead, the results are consistent with the view that the N2 in go/no-go tasks reflects conflict arising from competition between the execution and the inhibition of a single response.
\end{abstract}

Dealing with crosstalk, or conflict, in information processing is thought to be a central function of cognitive control (Allport, 1980, 1987; Norman \& Shallice, 1986). Recently, it has been suggested that the anterior cingulate cortex (ACC) may play an important role in monitoring for the occurrence of conflict during response selection (Botvinick, Braver, Barch, Carter, \& Cohen, 2001; Carter et al., 1998). According to this hypothesis, response conflict occurs whenever two or more incompatible response tendencies are simultaneously active. Upon the detection of such conflict, the ACC conveys a feedback signal to the brain areas involved in the actual execution of control (such as the prefrontal cortex), informing these areas that executive control processes must be

The research of S.N. was supported by the Netherlands Organization for Scientific Research (NWO). The research of K.R.R. was supported by the Royal Netherlands Academy of Arts and Sciences. We thank Jennifer Ramautar, Heleen Slagter, Zoe Tieges, and Boris Burle for technical, methodological, and logistic assistance and Jonathan Cohen for helpful comments on an earlier draft of the manuscript. We are grateful to Albert Kok for making available lab facilities at the University of Amsterdam. Correspondence concerning this article should be addressed to S. Nieuwenhuis, Department of Cognitive Psychology, Vrije Universiteit, Van der Boechorststraat 1, 1081 BT Amsterdam, The Netherlands (e-mail: stn20@dds.nl). more strongly engaged to prevent future conflict. The conflict-monitoring hypothesis can account for the finding that the ACC is reliably activated when subjects must overcome interference from an incorrect but prepotent response tendency (e.g., Botvinick, Nystrom, Fissell, Carter, \& Cohen, 1999; Carter et al., 2000), when they must choose between multiple response alternatives (Barch, Braver, Sabb, \& Noll, 2000), when they make response errors (Carter et al., 1998; Menon, Adleman, White, Glover, \& Reiss, 2001; Yeung, Botvinick, \& Cohen, 2001), and in other contexts in which the need for increased executive control becomes evident (see Botvinick et al., 2001, for a review).

Response conflict should be large when a low-frequency response must be made in a context of producing stereotyped or habitual responses: For the low-frequency response to be executed, it must compete with and eventually overcome the bias toward producing the prepotent response tendency (Braver, Barch, Gray, Molfese, \& Snyder, 2001; Jones, Cho, Nystrom, Cohen, \& Braver, 2002). The conflict caused by the simultaneous activation of the two competing response tendencies should result in increased ACC activity. In a recent neuroimaging study, Braver et al. (2001) have found support for this prediction. They found heightened ACC activity associ- 
ated with responding to low-frequency stimuli, as compared with responding to events occurring with high frequency. Importantly, the study included tasks involving competition between two alternative responses, as well as tasks involving competition between the generation and the suppression of a single response, suggesting that the frequency-dependent modulation of ACC activity occurs irrespective of the nature of the competing response tendencies.

Here, we are concerned with a task involving competition between generating and withholding a response: the go/no-go task. This task requires subjects to give a speeded, simple response to target (go) stimuli and to withhold any response to distractor (no-go) stimuli. The go/no-go task has been widely employed in human electrophysiological research. This research has led to the identification of an event-related brain potential (ERP), labeled the N2 (or no-go N2), that is strongly enhanced on no-go trials, as compared with go trials (e.g., Eimer, 1993; Jodo \& Kayama, 1992; Kok, 1986; Pfefferbaum, Ford, Weller, \& Kopell, 1985). The N2 can be observed as a negative shift over frontocentral scalp locations, with a peak between 250 and $350 \mathrm{msec}$ following the no-go stimulus. In previous research, the $\mathrm{N} 2$ enhancement on no-go trials (henceforth, the N2 effect) has been argued to reflect the operation of a cognitive top-down inhibition mechanism needed to suppress the incorrect tendency to respond. Early inhibition accounts assumed that the inhibition mechanism operated at the response execution level, serving to suppress motor activity on no-go trials. However, the finding that the N2 effect is not confined to overt motor responses but occurs also when subjects have to silently count go stimuli (Pfefferbaum et al., 1985) has led to the proposal that inhibition must be operating at a processing stage prior to motor execution (e.g., Falkenstein, Hoormann, \& Hohnsbein, 1999).

In this paper, we pursue the hypothesis that the increased N2 amplitude on no-go trials does not reflect the operation of an inhibitory process but, instead, represents an electrophysiological correlate of conflict monitoring by the ACC. This complements recent proposals that the N2 components associated with other tasks (such as the Eriksen flanker task) also reflect ACC sensitivity to response conflict (Kopp, Rist, \& Mattler, 1996; Van Veen \& Carter, 2002; Yeung et al., 2001). At first sight, the view that the $\mathrm{N} 2$ effect in the go/no-go task reflects differences in conflict on go and no-go trials (modulated by differences in trial type frequency) seems at odds with the common finding that the $\mathrm{N} 2$ effect is still present when go and no-go trials occur with equal frequency (e.g., Jodo \& Kayama, 1992; Kok, 1986). However, as we will argue in the Discussion section, the conflictmonitoring hypothesis can readily accommodate this finding by assuming that subjects' response tendency is biased toward the go response in go/no-go tasks.

A central assumption of our hypothesis is that no-go "responses" compete and conflict with internal representations of overt responses. Jones et al. (2002) have shown that a connectionist model implementing a conflict de- tection mechanism based on the competition between go and no-go response representations can account for behavioral and fMRI findings in the go/no-go task. Although somewhat counterintuitive, the notion of competing go and no-go response representations is supported by the results from a recent primate study of response inhibition in an oculomotor stop paradigm (Stuphorn, Taylor, \& Schall, 2000). In this study, the activation level of medial frontal cortex neurons (in the supplementary eye field) was found to be directly related to the degree of coactivation of gaze-shifting (i.e., go) and gaze-holding (no-go) neurons. We speculate that in manual response tasks, the no-go representation may correspond to a neural ensemble coding for a specific bodily posture (cf. Graziano, Taylor, \& Moore, 2002), which competes with representations of other postures.

In the present study, we explored some of the properties of the go/no-go N2 to test our hypothesis that this component can be better explained by the conflict-monitoring hypothesis than by the previously proposed inhibition hypothesis. We recorded high-density ERPs from subjects performing a go/no-go task in which the relative frequency of no-go trials $(20 \%, 50 \%$, or $80 \%)$ was varied across blocks of trials. We tested two predictions of the conflict-monitoring hypothesis, both of which were contrasted with the predictions of the inhibition hypothesis.

The first prediction of the conflict-monitoring hypothesis was that N2 amplitude should vary as a function of the relative frequency of go and no-go trials. That is, we expected that a progressive increase in the relative frequency of no-go trials should lead to a reduction and, eventually, even to a reversal of the $\mathrm{N} 2$ effect, so that the $\mathrm{N} 2$ should be enhanced on go trials in the condition in which these trials were rare. ${ }^{1}$ The inhibition hypothesis would be able to account for the expected initial reduction of the N2 effect by assuming that less inhibition is needed to prevent the execution of the go response on a no-go trial when the representation of the go response has a weaker baseline level of readiness. However, the inhibition hypothesis cannot account for a possible reversal of the $\mathrm{N} 2$ effect on infrequent go trials, because it predicts that no inhibition is required on go trials.

Motivated by the hypothesis that the N2 effect reflects conflict monitoring by the ACC, our second prediction was that the scalp distribution of the $\mathrm{N} 2$ effect should be consistent with a dipole source in the ACC region. In contrast, more lateral frontal areas are typically thought to be responsible for response inhibition (for a review, see Band \& van Boxtel, 1999). Although there have been several reports of no-go-specific field potential activity in the monkey lateral prefrontal cortex (e.g., Sasaki \& Gemba, 1986; Sasaki, Gemba, \& Tsujimoto, 1989), the early latency of this activity $(85-150 \mathrm{msec}$ following the no-go stimulus) suggests that it is not involved in the generation of the $\mathrm{N} 2$. We used inverse dipole modeling to test our hypothesis regarding the source of the N2. A recent high-density ERP study-in which frequent go and infrequent no-go stimuli were presented in a rapid serial visual presentation paradigm - showed initial evidence 
in line with our prediction (Bokura, Yamaguchi, \& Kobayashi, 2001). The N2 effect found in this study was localized to a neural source including the right ACC. We attempted to replicate and extend this result by examining whether the $\mathrm{N} 2$ effect could be localized to the same source as another ERP component, the error-related negativity (ERN [Gehring, Goss, Coles, Meyer, \& Donchin, 1993], or Ne [Falkenstein, Hohnsbein, Hoormann, \& Blanke, 1991]). The ERN is a negative component, similar in morphology to the $\mathrm{N} 2$, which is elicited following errors in speeded response time (RT) tasks (for a review, see Falkenstein, Hoormann, Christ, \& Hohnsbein, 2000). Recently, the ERN has been argued to represent an electrophysiological manifestation of response conflict on error trials (Botvinick et al., 2001; Yeung et al., 2001; for an alternative view, see Holroyd \& Coles, 2002) and has been localized in or very near the ACC (Dehaene, Posner, \& Tucker, 1994; Holroyd, Dien, \& Coles, 1998). Neuroimaging studies (e.g., Carter et al., 1998; Kiehl, Liddle, \& Hopfinger, 2000) have provided corroborating evidence for the activation of the ACC in association with errors. Thus, we expected that dipole modeling should result in the colocalization of the $\mathrm{N} 2$ effect and the ERN in the ACC.

\section{METHOD}

\section{Subjects}

Twelve undergraduate students ( 9 women) from the University of Amsterdam participated in a single 3-h session for course credit. The subjects ranged in age from 18 to 24 years ( $M$ age $=20.9$ years) and had normal or corrected-to-normal vision.

\section{Stimuli}

The stimuli were presented in red against a white background on a computer screen placed at a distance of roughly $90 \mathrm{~cm}$ from the subject. The stimuli were presented below a centrally presented black fixation dot, subtending $0.3^{\circ}$, which remained on the screen during the whole trial. The stimuli (either $\mathrm{M}$ or $\mathrm{W}$ in an uppercase Arial font) were presented for $100 \mathrm{msec}$, approximately $0.5^{\circ}$ below the fixation dot. The stimuli subtended $0.5^{\circ}$ vertically and $0.7^{\circ}$ horizontally. The interval separating successive stimuli was one of five equiprobable durations $(1.1,1.3,1.5,1.7$, or $1.9 \mathrm{sec})$.

\section{Design and Procedure}

There were three different task conditions. In the $20 \%$ no-go condition, the subjects were instructed to withhold responding to infrequent no-go stimuli ( $20 \%$ frequency) in the context of responding to frequent go stimuli ( $80 \%$ frequency). In the $80 \%$ no-go condition, the subjects were instructed to generate a response to infrequent go stimuli ( $20 \%$ frequency) in the context of withholding responses to frequent no-go stimuli. In the $50 \%$ no-go condition, go and no-go stimuli each occurred with $50 \%$ frequency.

The subjects received 100 practice trials with the $50 \%$ no-go condition before entering the experimental phase, which consisted of 12 blocks of 200 trials each. The order of task conditions across these 12 blocks was AABBCC CCBBAA (i.e., with the order of conditions during the second half of the experiment being the reverse of that during the first half of the experiment). Two subjects were assigned to each of the six possible orders. Response finger and the assignment of stimuli ( $\mathrm{M}$ and $\mathrm{W}$ ) to response options (go and no-go) were changed halfway through the experimental phase. The order of these two factors was varied orthogonally across subjects. During each block, go and no-go stimuli were presented in a randomly intermixed fashion (subject to the blockwise frequency ratios). There was a 15-min break halfway through the experimental phase and 2- to 3-min breaks between the other blocks. The subjects were informed about the frequency ratios in advance of each block and were instructed to press a response button "as quickly as possible" on go trials. Also, they were given feedback at the end of each block, showing their mean RT and error rate in that block.

\section{Psychophysiological Recording and Data Analysis}

EEG recordings were taken from 64 tin electrodes placed in an extended 10-20 system montage referenced to the left earlobe. The electro-oculogram (EOG) was recorded from tin electrodes placed above and below the left eye and from electrodes lateral to each eye. Electrode $\mathrm{AFz}$ served as the ground electrode. All electrode impedances were kept below $10 \mathrm{k} \Omega$. The EEG signals were digitized at $250 \mathrm{~Hz}$.

Single trial epochs were extracted off line and then corrected for EOG artifacts, using the algorithm described by Woestenburg, Verbaten, and Slangen (1983). Trials with RTs under $150 \mathrm{msec}$ and trials with recording artifacts were discarded $(4.8 \%)$. A prestimulus period of $100 \mathrm{msec}$ was subtracted as a baseline. For each participant and each condition, the EEG epochs were averaged with respect to both stimulus onset and response onset to obtain stimuluslocked and response-locked ERPs. The N2 and ERN amplitudes were computed on the basis of the signals obtained from $\mathrm{FCz}$, where both components were largest. In the stimulus-locked ERPs, the $\mathrm{N} 2$ was determined by searching backward in time for the first negative peak encountered in the window of 200-380 msec following the stimulus. N2 amplitude was defined as the amplitude of this peak minus the amplitude of the immediately preceding positive peak. In the response-locked ERPs, N2 amplitude was defined as the average value of the signal in the window $150-50 \mathrm{msec}$ preceding the response. ERN amplitude was defined as the most negative value of the signal in the window $0-150 \mathrm{msec}$ following the response minus the average value of the signal in the window 150$50 \mathrm{msec}$ preceding the response. Behavioral and ERP data were analyzed using repeated measures analyses of variance (ANOVAs), with Greenhouse-Geisser correction applied for violations of the ANOVA assumption of sphericity.

For the source localization analyses, the data from all 64 electrodes were 1-12 Hz bandpass filtered. Dipole models were then computed using BESA 2000 (www.besa.de) separately for the N2 difference waveform (no-go - go) in each of the three conditions and for the ERN difference waveform (error - correct) in the 20\% nogo condition, the condition with the most false alarms. Modeling was performed on data rereferenced to the average reference across a 16-msec window around the component peaks, using a three-shell spherical head model. Energy was included as a criterion to be minimized in fitting (i.e., along with residual variance). The reported dipole solutions were stable across different starting positions.

\section{RESULTS}

\section{Effects of Frequency on Performance and the N2}

Table 1 shows behavioral data for each trial type and each condition. ANOVAs confirmed that increasing the frequency of no-go trials led to an increased RT in response to go stimuli $[F(2,22)=113.9, p<.001]$ and to a decreased percentage of false alarms in response to nogo stimuli $[F(2,22)=87.5, p<.001]$. Performance accuracy on go trials was nearly perfect, owing to the fact that an initially withheld response could still be followed by the required buttonpress.

Figure 1 (left panel) shows the ERP waveforms associated with correct go trials and correct rejections in 
Table 1

$\underline{\text { Behavioral Performance Data as a Function of Trial Type and Task Condition }}$

\begin{tabular}{|c|c|c|c|c|c|c|c|c|}
\hline \multirow[b]{3}{*}{ Task Condition } & \multicolumn{4}{|c|}{ Go Trials } & \multicolumn{4}{|c|}{ No-Go Trials } \\
\hline & \multicolumn{2}{|c|}{$\overline{\mathrm{RT}}(\mathrm{msec})$} & \multicolumn{2}{|c|}{$\overline{\% \text { of Misses }}$} & \multicolumn{2}{|c|}{ FA RT (msec) } & \multicolumn{2}{|c|}{$\%$ of FAs } \\
\hline & $M$ & $S E$ & $M$ & $S E$ & $M$ & $S E$ & $M$ & $S E$ \\
\hline $20 \%$ no-go & 321 & 9 & 0.9 & 0.3 & 268 & 5 & 34.0 & 3.6 \\
\hline $50 \%$ no-go & 356 & 9 & 0.6 & 0.3 & 289 & 8 & 8.3 & 1.2 \\
\hline $80 \%$ no-go & 408 & 12 & 0.6 & 0.3 & - & - & 1.0 & 0.2 \\
\hline
\end{tabular}

Note-The percentage of false alarms (FAs) in the $80 \%$ no-go condition was too small to compute a reliable mean response time (RT) for these trials.

each of the three conditions. In the $20 \%$ no-go condition, $\mathrm{N} 2$ amplitude was larger on no-go trials $(-11.4 \mu \mathrm{V})$ than on go trials $(-4.5 \mu \mathrm{V})$. This $\mathrm{N} 2$ effect was reduced in the $50 \%$ no-go condition $(-6.3 \mu \mathrm{V}$ on no-go trials, $-4.5 \mu \mathrm{V}$ on go trials) and reversed in the $80 \%$ no-go condition $(-2.7 \mu \mathrm{V}$ vs. $-6.1 \mu \mathrm{V})$. The right panel of Figure 1 shows current source density (CSD) maps of the scalp topographies of the N2 effect (Perrin, Pernier, Bertrand, $\&$ Echallier, 1989). The data transformation underlying CSD maps serves as a high-pass frequency filter in the spatial domain and should, therefore, result in estimates of the N2 topographies that are relatively uncontaminated by the diffusely distributed and temporally overlapping P3 component. As is shown in Figure 1, the N2

\section{Stimulus-locked ERPs}
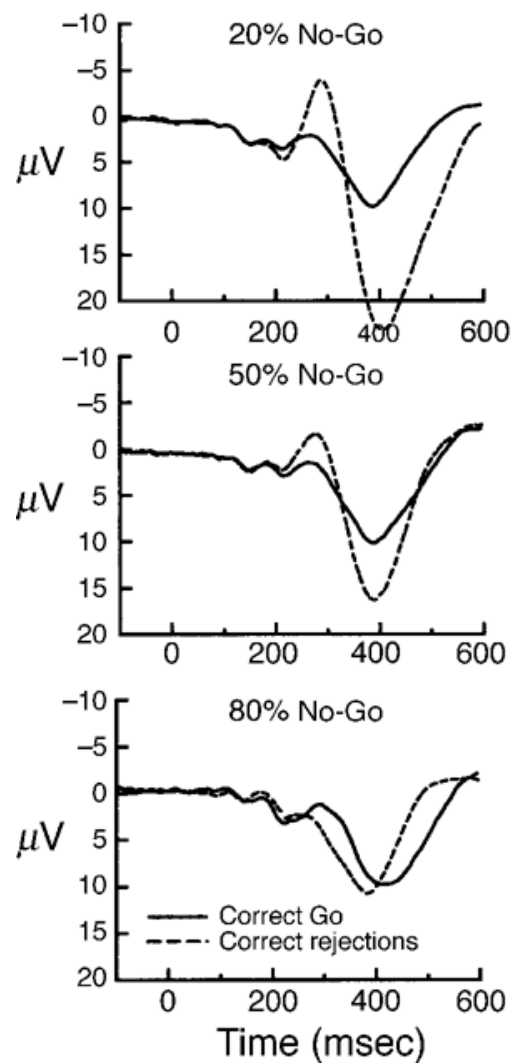

\section{CSD Difference Maps}
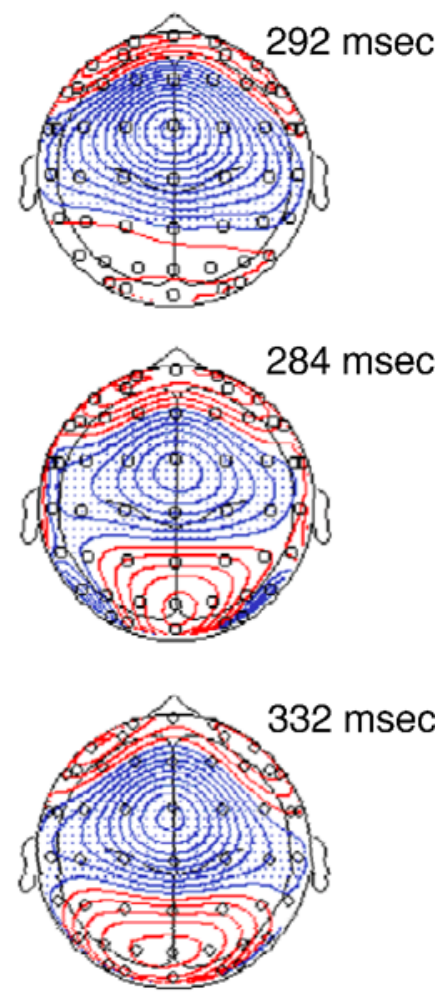

Figure 1. Left panel: stimulus-locked ERPs at electrode FCz for correct go trials and correct rejections, separated by task condition. Right panel: current source density (CSD) maps of the difference waves for correct trials (top and middle: no-go/go; bottom: go/no-go), representing the scalp topography of the $\mathrm{N} 2$ effect. Blue regions indicate negative values, red regions indicate positive values. The decrease/increase in CSD values represented by each isopotential line is $0.04 \mu \mathrm{V} / \mathrm{cm}^{2}$. In each of the three maps, the $\mathrm{N} 2$ effect is evident as a focal current sink at FCz. Maps were computed using BESA 2000. 
effect had a strikingly similar scalp topography across the three conditions, with peak amplitude at $\mathrm{FCz}^{2}$

N2 amplitudes for each subject were submitted to a 3 (condition) $\times 2$ (trial type) repeated measures ANOVA. The interaction of condition and trial type was highly significant $[F(2,22)=13.15, p=.002]$. The simple main effect of trial type was significant in each of the three conditions (all $p \mathrm{~s}<.05$ ). The simple main effect of condition for correct rejections was highly reliable $[F(2,22)=17.8$, $p<.001$ ], indicating that the amplitude of the $\mathrm{N} 2$ associated with correct rejections was inversely related to the relative frequency of no-go trials. Likewise, the amplitude of the N2 associated with correct go trials was inversely related to the relative frequency of go trials, but this relationship was weaker and failed to attain statistical significance $[F(2,22)=1.9, p=.19]$.

We hypothesized that the effect of condition on the go N2 should be more evident in the response-locked ERP waveforms. First, according to the conflict-monitoring hypothesis, the timing of any conflict-related ERP activity on correct trials should closely precede the timing of the response (Yeung et al., 2001). Interestingly, Ritter and colleagues (Ritter, Simpson, Vaughan, \& Friedman, 1979) have provided convincing evidence that the N2 on infrequent go trials precedes and covaries with the latency of the response. Second, it is possible that the effect of condition on the go N2 was somewhat obscured owing to differential overlap, across the conditions, with stimulus-related ERP components such as the P3. The response-locked ERPs for correct go trials, shown in
Figure 2 (left panel), were consistent with our prediction: An N2 was apparent just prior to the response, its amplitude varying inversely with the frequency of the go response. This effect of condition on the go N2 amplitude was reliable $[F(2,22)=5.1, p=.03]$. As was expected, the scalp distribution of this effect (defined as the difference in go $\mathrm{N} 2$ amplitude between the $20 \%$ no-go and the $80 \%$ no-go conditions) showed a maximal negativity over FCz (Figure 2, right panel).

\section{Dipole Modeling of N2 and ERN}

To test the prediction that the N2 and the ERN shared a similar neural source in or near the ACC, we computed and compared dipole source models of the observed scalp voltage distributions for the N2 and ERN difference waves (see the Method section). We focused our analysis on the $20 \%$ no-go condition, because (1) the N2 effect was largest in this condition and (2) in this condition the number of errors was sufficient to compute a reliable ERN difference wave. Figure 2 allows a comparison between the response-locked ERPs for correct go trials and the false alarms in the $20 \%$ no-go condition. The ERN is clearly evident as a negative deflection in the waveform for false alarms, starting just prior to the response and peaking around $70 \mathrm{msec}$ after the response. The difference in amplitude between correct go trials and false alarms was reliable $[F(1,11)=10.0, p=.009]$.

Figure 2 (right panel) confirms that the scalp topography of the ERN was similar to that of the N2, with peak amplitude at FCz. Furthermore, as is shown in Figure 3,

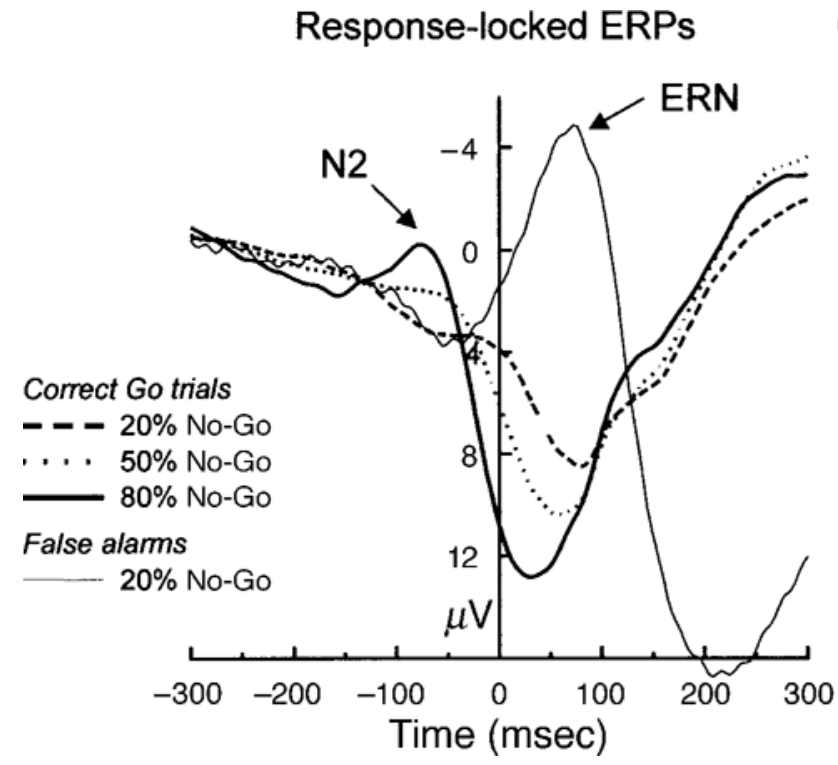

CSD Difference Maps
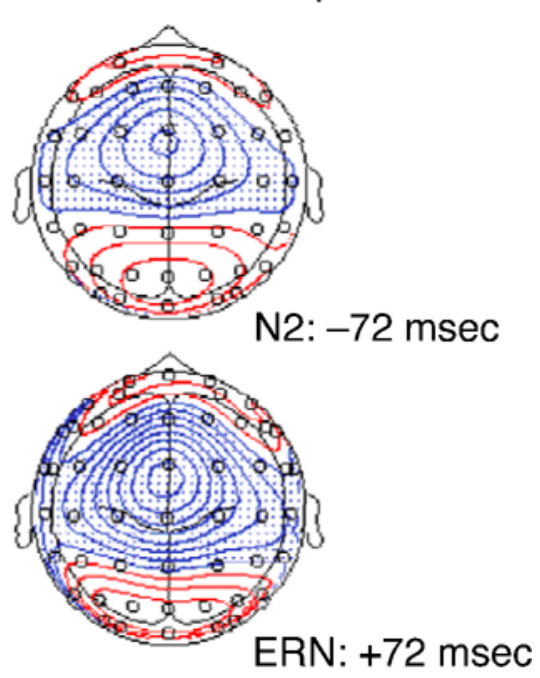

Figure 2. Left panel: response-locked ERPs at electrode FCz for correct go trials for each task condition and for false alarms in the $20 \%$ no-go condition. Time $=0 \mathrm{msec}$ indicates the timing of the response. The $\mathbf{N} 2$ effect, prior to the response, and the ERN, following the response, are clearly visible. Right panel: current source density (CSD) maps of the response-locked difference waves representing the scalp topography of the $\mathrm{N} 2$ effect on correct go trials $(80 \%$ no-go - $20 \%$ no-go) and the ERN in the $20 \%$ no-go condition (false alarms - correct go trials). Blue regions indicate negative values; red regions indicate positive values. The decrease/increase in CSD values represented by each isopotential line is $0.07 \mu \mathrm{V} / \mathrm{cm}^{2}$. The $\mathrm{N} 2$ and the ERN are both evident as a focal current sink at $\mathrm{FCz}$. 


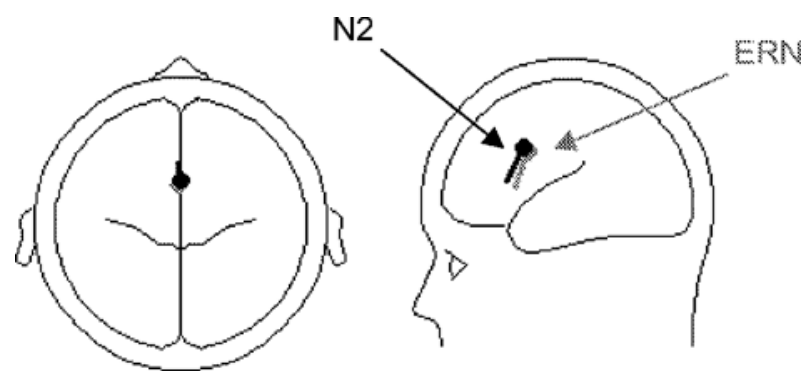

Figure 3. Single-dipole models of the $\mathrm{N2}$ effect and the errorrelated negativity (ERN) effect in the $20 \%$ no-go condition. See the text for further information.

the best-fitting dipoles for the $\mathrm{N} 2$ and the ERN lay very close together in the medial frontal cortex, consistent with a common neural source in the ACC. The single-dipole models explained most of the variance in the data for the $\mathrm{N} 2(x=-0.1 \mathrm{~cm}, y=2.5 \mathrm{~cm}, z=7.0 \mathrm{~cm} ; \mathrm{RV}=5.5 \%)$ and for the ERN ( $x=-0.1 \mathrm{~cm}, y=2.2 \mathrm{~cm}, z=6.7 \mathrm{~cm}$; RV = $6.6 \%)$ difference waves. To test the possibility of $\mathrm{N} 2$ sources in the lateral prefrontal cortex, we evaluated whether bilateral symmetric dipole models could account for the observed scalp distribution. We tested three specific models, for which the locations of the dipole pairs corresponded roughly to Brodmann areas 9, 44, and 46 in the lateral prefrontal cortex, three areas often reported in fMRI studies of go/no-go-related brain activity (e.g., Braver et al., 2001; Menon et al., 2001). The orientations of each dipole pair were allowed to vary freely. The three models showed substantially worse fits than the reported single-dipole model: area $9(x=-3.9 \mathrm{~cm}$, $y=3.5 \mathrm{~cm}, z=7.5 \mathrm{~cm}$; coordinates correspond to the left-hemisphere dipole; $\mathrm{RV}=9.7 \%$; energy $=17.4)$; area $44(x=-5.1 \mathrm{~cm}, y=2.0 \mathrm{~cm}, z=5.5 \mathrm{~cm}$; RV $=13.4 \%$; energy $=18.1)$; area $46(x=-3.9 \mathrm{~cm}, y=5.0 \mathrm{~cm}, z=$ $5.5 \mathrm{~cm} ; \mathrm{RV}=23.2 \%$; energy $=15.0$ ). Further exploration of sources in the lateral prefrontal cortex yielded no satisfactory symmetric dipole solutions. Moreover, when we took the three dipole pairs as starting positions for the fitting procedure and then allowed the dipole locations and orientations to vary freely, the dipoles migrated to symmetrical locations in the medial frontal cortex, very close to the reported single-dipole source.

We also explored source models of the N2 in the $50 \%$ no-go condition and the $80 \%$ no-go condition, although the smaller sizes of the $\mathrm{N} 2$ effect decreased the chances of finding a reliable dipole solution. In the $50 \%$ no-go condition, the best-fitting single dipole was a very deep and implausible source in the brainstem. This dipole explained almost all of the variance $(>96 \%)$ and was stable to the addition of another freely varying dipole. Source analysis seemed uninformative in this condition. In the $80 \%$ no-go condition, the best-fitting single dipole, roughly located in the medial superior frontal gyrus, had an unsatisfactory goodness of fit $(\mathrm{RV}=10.5 \%)$. In contrast, an unconstrained two-dipole model led to a good fit $(\mathrm{RV}=3.2 \%$; energy $=55.9)$. This model, shown in Figure 4, involved one dipole in the ACC $(x=0.1 \mathrm{~cm}, y=$ $2.6 \mathrm{~cm}, z=6.7 \mathrm{~cm})$ and one dipole in the medial posterior cortex $(x=0.8 \mathrm{~cm}, y=-5.1 \mathrm{~cm}, z=5.5 \mathrm{~cm})$. The ACC dipole virtually overlapped with the single dipole in the $20 \%$ no-go condition. Furthermore, the source, the orientation, and the moment (late and protracted relative to the ACC dipole) of the posterior dipole suggest that it may model P3 activity. This appears to be consistent with the finding that in the $80 \%$ no-go condition, the N2 was superimposed on the P3.

\section{DISCUSSION}

The present research was motivated by previous studies reporting a negative ERP component, the N2, whose amplitude is sensitive to the response requirements in a go/no-go task (e.g., Eimer, 1993; Jodo \& Kayama, 1992; Kok, 1986; Pfefferbaum et al., 1985). We hypothesized that the go/no-go N2 reflects an electrophysiological correlate of conflict between the go and no-go response representations that is detected in the ACC. This hypothesis was motivated by recent research suggesting a role for the ACC in monitoring for response conflict in a variety of tasks, including go/no-go tasks (e.g., Botvinick et al., 2001; Braver et al., 2001). Response conflict should be substantial under conditions in which a lowfrequency response is required in the context of making other, high-frequency responses. Thus, we predicted that the $\mathrm{N} 2$ in the go/no-go task should be enhanced in the ERP elicited by stimuli occurring with a low frequency, irrespective of whether these stimuli were associated with a go or a no-go response. We also predicted that the $\mathrm{N} 2$ should have a neural source in the ACC. To test these predictions, we collected high-density ERP data from subjects performing a go/no-go task in which the relative frequency of go versus no-go stimuli was varied. Consistent with our predictions, we observed a reliable $\mathrm{N} 2$ enhancement associated with infrequent go and nogo stimuli. Furthermore, inverse dipole modeling suggested a common source in the ACC of the N2 and of the ERN, an ERP component that has been interpreted in terms of conflict monitoring on error trials (Carter et al., 1998; Yeung et al., 2001).
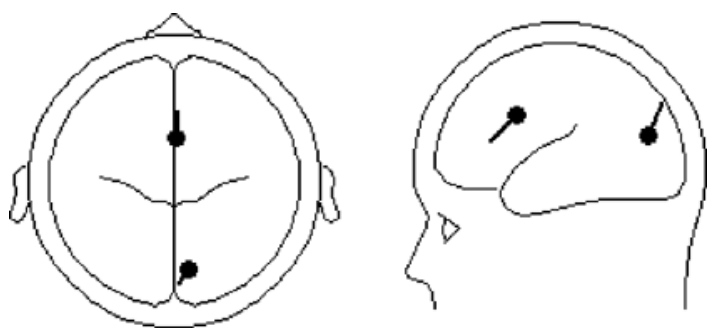

Figure 4. Two-dipole model of the N2 effect in the $80 \%$ no-go condition. 
Taken at face value, the conflict-monitoring hypothesis predicts that $\mathrm{N} 2$ amplitude should be the same on go and no-go trials when they occur with equal frequency. Contrary to this prediction, however, we found that the $\mathrm{N} 2$ was larger on no-go trials than on go trials even in the 50\% no-go condition. Moreover, the no-go N2 was much larger than the go N2 in the $20 \%$ no-go condition, whereas the go $\mathrm{N} 2$ was only slightly larger than the no-go N2 in the $80 \%$ no-go condition. To explain these results in terms of conflict monitoring, we must therefore make one additional, although plausible, assumption: that the requirement to respond "as quickly as possible"-as emphasized, for instance, in the present task instructions-causes a general bias toward the go response (on top of any bias caused by relative trial type frequency). The additional bias toward the go response leads to increased conflict on no-go trials and to reduced conflict on go trials and could thus explain the asymmetry of the N2 effects. Therefore, we suggest that the asymmetry of the N2 effects is a direct consequence of the asymmetry in task instructions with respect to go and no-go trials.

This assumption granted, the conflict-monitoring hypothesis provides a common framework for explaining scalp negativities observed during the performance of a variety of experimental tasks. For instance, the N2 enhancement in response to infrequent go ("oddball") stimuli has typically been treated as a qualitatively different effect (and in a different part of the ERP literature) than the $\mathrm{N} 2$ enhancement in response to infrequent no-go stimuli. However, our results suggest that these effects have a common origin. That is, not only are they similar in timing and scalp distribution, but also they can be accounted for in terms of variation in a single variable: relative trial type frequency. The notion that conflict is modulated by the relative frequency of responses (Braver et al., 2001; Jones et al., 2002) presents an account of the specific mechanism by which this variable affects brain activity. The conflict model also provides a natural explanation for the observation of very similar ERP components in other tasks thought to elicit response conflict, such as the Eriksen flanker task. The timing, scalp distribution, and supposed neural source of the $\mathrm{N} 2$ observed in the flanker task are very similar to those of the N2 component studied here (Heil, Osman, Wiegelmann, Rolke, \& Hennighausen, 2000; Kopp, Rist, \& Mattler, 1996; Van Veen \& Carter, 2002; Yeung et al., 2001).

The conflict-monitoring hypothesis of the N2 can explain several further findings in the go/no-go ERP literature that have previously been interpreted in terms of the inhibition hypothesis. Given that conflict is assumed to occur at the level of response representations, it follows that the $\mathrm{N} 2$ should also be sensitive to competition between covert responses. This explains the finding that similar N2 modulations are observed when the required go response is to count stimuli, rather than to press a button (Pfefferbaum et al., 1985). The conflict-monitoring hypothesis also provides a natural explanation for the finding that the no-go N2 increases if the go response is transiently primed by a cue preceding the stimulus ${ }^{3}$ (Kopp, Mattler, Goertz, \& Rist, 1996) or is at a higher baseline level of activation owing to the requirement to meet a stringent RT deadline on go trials (Jodo \& Kayama, 1992). This is a straightforward consequence of the increased bias toward the go response, yielding higher response conflict when the no-go response representation must compete with and overcome the activation of the go response.

A result reported by Falkenstein et al. (1999) appears to challenge the conflict-monitoring hypothesis. These authors divided their subjects into two groups, according to a median split on the basis of false alarm rates, and found that the "good performers" showed a reliably larger N2 than did the "poor performers." This finding appears to raise problems for the conflict-monitoring hypothesis, because it suggests that good performance is associated with large response conflict. In contrast, it can readily be explained by the inhibition hypothesis (i.e., good performance is associated with stronger inhibition of the incorrect response). However, given the correlational nature of this result, interpretations consistent with the conflict-monitoring hypothesis also seem plausible. Most notably, a third variable (i.e., general efficiency of monitoring and regulation of performance) may have influenced both the strength of the conflictmonitoring signal (N2) and the percentage of correctly inhibited responses in such a way that these two were positively correlated across subjects.

The present findings cannot easily be explained by theories that associate the $\mathrm{N} 2$ with response inhibition. Inhibition accounts may explain why $\mathrm{N} 2$ amplitude on no-go trials is reduced as the relative frequency of no-go trials is increased. However, these theories cannot explain why an N2 is observed on go trials when such trials are rare, since no inhibition should be required on those trials. It may be possible to develop more sophisticated inhibition accounts to deal with this finding. For example, one might propose that the $\mathrm{N} 2$ reflects two separate processes: top-down inhibition and an attentional "mismatch" process that is sensitive to infrequent physical deviations from the prevailing context (see Mäntysalo, 1987). This account can explain why N2 amplitude is usually greater on no-go than on go trials (since inhibition is required only on the former) and why a small N2 is nonetheless observed on go trials when such trials are rare (since these trials deviate from the expectation of the frequent no-go trials). However, apart from the need to invoke two separate processes, this account requires the strong assumption that these two processes are reflected in two ERP components that are similar in terms of latency, morphology, and scalp distribution.

One might alternatively propose that top-down inhibition is also needed on (rare) go trials to inhibit a prepotent no-go response tendency. The similarity between the N2 components observed on go and no-go trials would be 
consistent with this theory. However, top-down inhibition is often associated with the functions of the lateral prefrontal cortex (e.g., Jonides, Smith, Marshuetz, Koeppe, \& Reuter-Lorenz, 1998; for a review, see Band \& van Boxtel, 1999), and a wealth of neuroimaging evidence suggests that the ACC's role in cognitive control is primarily evaluative in nature (see, e.g., Barch et al., 2000; Botvinick et al., 1999; Bush, Luu, \& Posner, 2000). Thus, the localization of the source of the N2 in the ACC raises problems for inhibition theories. The colocalization of the N2 with the ERN — an electrophysiological marker of a clearly evaluative process-is further suggestive of the notion that the $\mathrm{N} 2$ indexes an evaluative function. ${ }^{4} \mathrm{In}$ particular, it provides further support for the hypothesis that these components represent different manifestations of response conflict (Van Veen \& Carter, 2002; Yeung et al., 2001). As was demonstrated by Yeung et al., response conflict should closely precede the response on correct trials and immediately follow the response on incorrect trials. The timing of the N2 and the ERN in our study (see Figure 2) is in line with this view.

Finally, if the N2 reflects ACC activity, this may have consequences for theories of ACC function. We have argued that the conflict-monitoring hypothesis can account for the available $\mathrm{N} 2$ data, but there exist other broad theories of ACC function. One prominent theory holds that the ACC is involved in the selection of motor control systems relevant for the task at hand (e.g., Holroyd \& Coles, 2002; Paus, Petrides, Evans, \& Meyer, 1993). According to another prominent theory, the rostral-ventral ACC is a part of a circuit involved in the regulation of emotional processing (see, e.g., Bush et al., 2000), leading to the suggestion that the ERN may reflect the emotional evaluation of an error in the ACC (e.g., Gehring, Himle, \& Nisenson, 2000). An interesting question that is open for future debate is whether these theories of ACC function are able to account for the N2 results reported here and elsewhere.

In conclusion, we suggest that the $\mathrm{N} 2$ observed in go/no-go tasks reflects response conflict monitoring by the ACC. The present results indicate that the $\mathrm{N} 2$ observed in go/no-go tasks is reliably enhanced by the requirement to respond to low-frequency stimuli, irrespective of whether these stimuli are associated with a go or a no-go response. Furthermore, the source of the N2 was localized to the ACC, as was the source of the ERN. We have suggested that these findings cannot be readily explained in terms of the response inhibition hypothesis previously thought to account for the go/no-go N2. Instead, the results are consistent with the hypothesis proposed here, that the go/no-go N2 represents an ERP correlate of response conflict monitoring on correct trials. This view provides a unifying account of the $\mathrm{N} 2$ components observed in a variety of experimental tasks and fits with a growing literature that links ACC activity to evaluative aspects of cognitive control (e.g., Botvinick et al., 2001; Carter et al., 1998; Ridderinkhof, Nieuwenhuis, \& Bashore, 2002).

\section{REFERENCES}

Allport, D. A. (1980). Attention and performance. In G. Claxton (Ed.), Cognitive psychology: New directions (pp. 112-153). London: Routledge \& Kegan Paul.

Allport, D. A. (1987). Selection for action: Some behavioural and neurophysiological considerations of attention and action. In H. Heuer \& A. F. Sanders (Eds.), Perspectives on perception and action (pp. 395-419). Hillsdale, NJ: Erlbaum.

BAND, G. P. H., \& VAN Boxtel, G. J. M. (1999). A cognitive-neuroscience perspective on the locus of response inhibition. Acta Psychologica, 101, 315-337.

Barch, D. M., Braver, T. S., SABb, F. W., \& Noll, D. C. (2000). Anterior cingulate and the monitoring of response conflict: Evidence from an fMRI study of overt verb generation. Journal of Cognitive Neuroscience, 12, 298-309.

Bokura, H., Yamaguchi, S., \& Kobayashi, S. (2001). Electrophysiological correlates for response inhibition in a Go/NoGo task. Clinical Neurophysiology, 112, 2224-2232.

Botvinick, M. M., Braver, T. S., Barch, D. M., Carter, C. S., \& Cohen, J. D. (2001). Conflict monitoring and cognitive control. Psychological Review, 108, 624-652.

Botvinick, M. M., Nystrom, L. E., Fissell, K., Carter, C. S., \& CoHen, J. D. (1999). Conflict monitoring versus selection-for-action in anterior cingulate cortex. Nature, 402, 179-181.

Braver, T. S., Barch, D. M., Gray, J. R., Molfese, D. L., \& Snyder, A. (2001). Anterior cingulate cortex and response conflict: Effects of frequency, inhibition and errors. Cerebral Cortex, 11, 825-836.

Bush, G., LuU, P., \& Posner, M. I. (2000). Cognitive and emotional influences in anterior cingulate cortex. Trends in Cognitive Sciences, $\mathbf{4}$, $215-222$.

Carter, C. S., Braver, T. S., Barch, D. M., Botvinick, M. M., Noll, D., \& Cohen, J. D. (1998). Anterior cingulate cortex, error detection, and the online monitoring of performance. Science, 280, 747-749.

Carter, C. S., MacDonald, A. M., III, Botvinick, M. M., Ross, L. L., Stenger, V. A., Noll, D., \& Cohen, J. D. (2000). Parsing executive processes: Strategic vs. evaluative functions of the anterior cingulate cortex. Proceedings of the National Academy of Sciences, 97, 1944-1948.

Czigler, I., Csibra, G., \& Ambro, A. (1996). Aging, stimulus identification and the effect of probability: An event-related potential study. Biological Psychology, 43, 27-40.

Dehaene, S., Posner, M. I., \& Tucker, D. M. (1994). Localization of a neural system for error detection and compensation. Psychological Science, 5, 303-305.

EIMER, M. (1993). Effects of attention and stimulus probability on ERPs in a Go/Nogo task. Biological Psychology, 35, 123-138.

Falkenstein, M., Hohnsbein, J., Hoormann, J., \& Blanke, L. (1991). Effects of crossmodal divided attention on late ERP components: II. Error processing in choice reaction tasks. Electroencephalography \& Clinical Neurophysiology, 78, 447-455.

Falkenstein, M., Hoormann, J., Christ, S., \& Hohnsbein, J. (2000). ERP components on reaction errors and their functional significance: A tutorial. Biological Psychology, 51, 87-107.

Falkenstein, M., Hoormann, J., \& Hohnsbein, J. (1999). ERP components in Go/Nogo tasks and their relation to inhibition. Acta Psychologica, 101, 267-291.

Gehring, W. J., Goss, B., Coles, M. G. H., Meyer, D. E., \& Donchin, E (1993). A neural system for error detection and compensation. Psychological Science, 4, 385-390.

Gehring, W. J., Himle, J., \& Nisenson, L. G. (2000). Action-monitoring dysfunction in obsessive-compulsive disorder. Psychological Science, 11, 1-6.

Graziano, M. S. A., Taylor, C. S. R, \& Moore, T. (2002). Complex movements evoked by microstimulation of precentral cortex. $\mathrm{Neu}$ ron, 34, 841-851.

Heil, M., Osman, A. Wiegelmann, J., Rolke, B., \& Hennighausen, E. (2000). N200 in the Eriksen-paradigm: Inhibitory executive processes? Journal of Psychophysiology, 14, 218-225.

Holroyd, C. [B.], \& Coles, M. G. H. (2002). The neural basis of 
human error processing: Reinforcement learning, dopamine, and the error-related negativity. Psychological Review, 109, 679-709.

Holroyd, C. B., Dien, J., \& Coles, M. G. H. (1998). Error-related scalp potentials elicited by hand and foot movements: Evidence for an outputindependent error-processing system in humans. Neuroscience Letters, 242, 65-68.

Jodo, E, \& KaYAma, Y. (1992). Relation of a negative ERP component to response inhibition in a go/no-go task. Electroencephalography \& Clinical Neurophysiology, 82, 477-482.

Jones, A. D., Cho, R. Y., Nystrom, L. E., Cohen, J. D., \& Braver, T. S. (2002). A computational model of anterior cingulate function in speeded response tasks: Effects of frequency, sequence, and conflict. Cognitive, Affective, \& Behavioral Neuroscience, 2, 300-317.

Jonides, J., Smith, E. E., Marshuetz, C., Koeppe, R. A., \& ReuterLORENZ, P. A. (1998). Inhibition in verbal working memory revealed by brain activation. Proceedings of the National Academy of Sciences, 95, 8410-8413.

Kiehl, K. A., Liddle, P. F., \& Hopfinger, J. B. (2000). Error processing and the rostral anterior cingulate: An event-related fMRI study. Psychophysiology, 37, 216-223.

KoK, A. (1986). Effects of degradation of visual stimulation on components of the event-related potential (ERP) in Go/NoGo reaction tasks. Biological Psychology, 23, 21-38.

Kopp, B., Mattler, U., Goertz, R. \& Rist, F. (1996). N2, P3 and the lateralized readiness potential in a nogo task involving selective response priming. Electroencephalography \& Clinical Neurophysiology, 99, 19-27.

Kopp, B., Rist, F., \& Mattler, U. (1996). N200 in the flanker task as a neurobehavioral tool for investigating executive control. Psychophysiology, 33, 282-294.

MäNTYSALO, S. (1987). N2 and P3 of the ERP to Go and Nogo stimuli: A stimulus-response association and dissociation. In R. Johnson, Jr., J. W. Rohrbaugh, \& R. Parasuraman (Eds.), Current trends in eventrelated potential research (Electroencephalography \& Clinical Neurophysiology, Suppl. 40, pp. 227-234). Amsterdam: Elsevier.

McCarthy, G., \& Wood, C. C. (1985). Scalp distributions of eventrelated potentials: An ambiguity associated with analysis of variance models. Electroencephalography \& Clinical Neurophysiology, 62, 203-208.

Menon, V., Adleman, N. E., White, C. D., Glover, G. H., \& Reiss, A. L. (2001). Error-related brain activation during a Go/NoGo response inhibition task. Human Brain Mapping, 12, 131-143.

NäÄTÄNEN, R. (1992). Attention and brain function. Hillsdale, NJ: Erlbaum.

Norman, D. A., \& Shallice, T. (1986). Attention to action: Willed and automatic control of behavior. In R. J. Davidson, G. E. Schwartz, \& D. Shapiro (Eds.), Consciousness and self-regulation (pp. 1-18). New York: Plenum.

Paus, T., Petrides, M., Evans, A. C., \& Meyer, E. (1993). Role of the human anterior cingulate cortex in the control of oculomotor, manual, and speech responses: A positron emission tomography study. Journal of Neurophysiology, 70, 453-469.

Perrin, F., Pernier, J., Bertrand, O., \& Echallier, J. F. (1989). Spherical splines for scalp potential and current density mapping. Electroencephalography \& Clinical Neurophysiology, 72, 184-187.

Pfefferbaum, A., Ford, J. M., Weller, B. J., \& Kopell, B. S. (1985). ERPs to response production and inhibition.Electroencephalography \& Clinical Neurophysiology, 60, 423-434.

Pritchard, W. S., Shappell, S. A., \& Brandt, M. E. (1991). Psychophysiology of N200/N400: A review and classification scheme. Advances in Psychophysiology, 4, 43-106.

Ridderinkhof, K. R., Nieuwenhuis, S., \& Bashore, T. R. (2002). Errors are foreshadowed in brain potentials associated with action monitoring in cingulate cortex. Manuscript submitted for publication.

Ritter, W., Simpson, R., Vaughan, H. G., \& Friedman, D. (1979). A brain event related to the making of a sensory discrimination. Science, 203, 1358-1361.

SASAKI, K., \& Gemba, H. (1986). Electrical activity in the prefrontal cortex specific to no-go reaction of conditioned hand movement with colour discrimination in the monkey. Experimental Brain Research, 64, 603-606

Sasaki, K., Gemba, H., \& Tsujimoto, T. (1989). Suppression of visually initiated hand movement by stimulation of the prefrontal cortex in the monkey. Brain Research, 495, 100-107.

SCHRÖGER, E. (1993). Event-related potentials to auditory stimuli following transient shifts of spatial attention in a Go/Nogo task. Biological Psychology, 36, 183-207.

Stuphorn, V., TAYlor, T. L., \& Schall, J. D. (2000). Performance monitoring by the supplementary eye field. Nature, 408, 857-860.

VAn Veen, V., \& CARTER, C. S. (2002). The timing of action monitoring processes in anterior cingulate cortex. Journal of Cognitive Neuroscience, 14, 593-602.

Woestenburg, J. C., Verbaten, M. N., \& Slangen, J. L. (1983). The removal of the eye-movement artifact from the EEG by regression analysis in the frequency domain. Biological Psychology, 16, 127147.

Yeung, N., Botvinick, M. M., \& Cohen, J. D. (2001). The neural basis of error detection: Conflict monitoring and the error-related negativity. Manuscript submitted for publication.

\section{NOTES}

1. There are fragments of evidence consistent with this prediction in the existing literature. First, there are some reports of an enhanced N2 effect when no-go stimuli constitute a low-frequency event, as compared with when they occur with higher probability. However, this evidence is based on between-experiment comparisons (Eimer, 1993; Schröger, 1993) and on visual examination of published data (Czigler, Csibra, \& Ambro, 1996). Nevertheless, it is at least consistent with the conflict-monitoring hypothesis. In addition, our hypothesis predicts that an N2 should be observed on go trials if they occur with relatively low frequency. Studies in which the classical oddball paradigm has been used, in which infrequent go stimuli are interspersed with frequent no-go stimuli, suggest that this is indeed the case (for reviews, see Näätänen, 1992, and Pritchard, Shappell, \& Brandt, 1991).

2. Unfortunately, we know of no straightforward way to quantify the topographies shown in CSD maps. The untransformed voltage maps, however, are amenable to straightforward statistical analysis. Therefore, even though CSD maps should be preferred over voltage maps, we statistically compared the original (untransformed) voltage distributions, normalized according to McCarthy and Wood (1985), by performing a condition $\times$ anterior/posterior electrode location $(\mathrm{F}, \mathrm{Fc}, \mathrm{C}, \mathrm{Cp}, \mathrm{P}) \times$ laterality $(3, z, 4)$ ANOVA. The condition $\times$ laterality interaction was not significant $[F(4,44)=1.6, p=.24]$. The condition $\times$ anterior/posterior interaction was significant $[F(8,88)=5.3, p=.013]$. Pairwise comparisons between the conditions indicated that the $\mathrm{N} 2$ effect in the $50 \%$ nogo condition had a significantly more anterior voltage distribution than did the other two conditions. This is clearly not the case in the CSD maps, suggesting that differential contributions of the P3 to these voltage distributions may underlie the difference between conditions. The $20 \%$ no-go condition and the $80 \%$ no-go condition did not differ from each other.

3. On a related point, the conflict-monitoring hypothesis makes a specif ic prediction about the effect of trial type of the previous trial on the N2 effect: The N2 enhancement for any trial type should be decreased if the associated response is primed on the previous trial. There were reliable sequential priming effects in the behavioral data: After no-go trials, the subjects responded more slowly on go trials $[F(1,11)=14.0, p=$ $.003]$ and made fewer false alarms on no-go trials $[F(1,11)=17.7, p=$ $.001]$ than after go trials. In the $50 \%$ no-go and $80 \%$ no-go conditions, there was a sufficient number of correct trials to compute reliable ERPs for each of the combinations of current trial type and previous trial type, so we confined our N2 analyses to these two conditions. As was expected, in the $50 \%$ no-go condition, the N2 enhancement on no-go trials was smaller on trials following a no-go trial $(-0.2 \mu \mathrm{V})$ than on trials following a go trial $(-3.9 \mu \mathrm{V})$, whereas in the $80 \%$ no-go condition, the $\mathrm{N} 2$ enhancement on go trials was less pronounced on trials following a go trial $(-1.5 \mu \mathrm{V})$ than on trials following a no-go trial $(-4.4 \mu \mathrm{V})$. 
However, this interaction between current trial type and previous trial type was not significant $\left[F(1,11)=2.4, M S_{\mathrm{e}}=16.0, p=.15\right]$, possibly owing to a lack of power (observed power $=.29$ ). Future research, incorporating a sufficient number of trials and subjects, is needed to reliably test predictions regarding sequential effects on $\mathrm{N} 2$ amplitude.

4. The colocalization of the ERN and the no-go N2 seems inconsistent with a recent study by Falkenstein and colleagues (Falkenstein et al., 1999), who reported that the go/no-go N2 and ERN have different scalp distributions, with the N2 being largest at Fz and the ERN being largest at $\mathrm{Cz}$. However, these authors did not record from $\mathrm{FCz}$, leaving open the possibility that both components were largest at this electrode site, as was the case in the present study. Moreover, visual in- spection of Falkenstein et al.'s (1999) response-locked ERP waveforms (Falkenstein et al., 1999, Figure 7) suggests that the difference in estimates of the ERN amplitudes at $\mathrm{Fz}$ and $\mathrm{Cz}$ is, at least in part, an artifact of the subtraction of errors from correct responses used to derive these estimates. That is, the electrode effect in the difference wave is caused mainly by the fact that the P3-like component in the correct waveforms is larger at $\mathrm{Cz}$ than at $\mathrm{Fz}$ (as is usually the case for the P3).

(Manuscript received April 30, 2002;

revision accepted for publication October 14, 2002.) 